\title{
Tackling Cannibalization Problems for Online Advertisement
}

\author{
Yutaro Ueoka \\ Yahoo Japan Corporation \\ yueoka@yahoo-corp.jp
}

\author{
Kota Tsubouchi \\ Yahoo Japan Corporation \\ ktsubouc@yahoo-corp.jp
}

\author{
Nobuyuki Shimizu \\ Yahoo Japan Corporation \\ nobushim@yahoo-corp.jp
}

\begin{abstract}
Market cannibalization is inevitable when there are two or more competing marketing approaches to the same customer base. The cannibalization problem has been discussed in the context of search advertising of individual advertisers, whereas in this paper we discuss the problem that advertising platform companies face in dealing with multiple advertisers. In online advertising, they must properly serve ads with varying mass appeal to users with various interests. For them, it is important to maximize the value of the ads for advertisers and also for the platform. To do so, they deploy user models to serve ads. However, shortsighted models could lead to a decrease in overall performance in an attempt to improve certain ads' performance while slightly impairing the rest. We consider this phenomenon from the perspective of cannibalization and confirm the existence of a cannibalization problem in optimizing the delivery of ads in minor categories. To resolve this problem, we propose new methods, apply them to an ad delivery system, and conduct an $\mathrm{A} / \mathrm{B}$ test. Our methods overcame the cannibalization problem and increased revenue by $+0.6 \%$ compared with the baseline method.
\end{abstract}

\section{CCS CONCEPTS}

- Information systems $\rightarrow$ Display advertising; Computational advertising; Personalization; • Computing methodologies $\rightarrow$ Modeling methodologies.

\section{KEYWORDS}

cannibalization, user modeling for online advertisement, online questionnaire survey

\section{ACM Reference Format:}

Yutaro Ueoka, Kota Tsubouchi, and Nobuyuki Shimizu. 2020. Tackling Cannibalization Problems for Online Advertisement. In Proceedings of the 28th ACM Conference on User Modeling, Adaptation and Personalization (UMAP '20), July 14-17, 2020, Genoa, Italy. ACM, New York, NY, USA, 5 pages. https://doi.org/10.1145/3340631.3394875

\section{INTRODUCTION}

Online advertising, which employs sophisticated models of user characteristics, excels at serving ads to specific and targeted audiences, which traditional offline mass media advertising has difficulty

Permission to make digital or hard copies of all or part of this work for personal or classroom use is granted without fee provided that copies are not made or distributed for profit or commercial advantage and that copies bear this notice and the full citation on the first page. Copyrights for components of this work owned by others than ACM must be honored. Abstracting with credit is permitted. To copy otherwise, or republish, to post on servers or to redistribute to lists, requires prior specific permission and/or a fee. Request permissions from permissions@acm.org.

UMAP '20, fuly 14-17, 2020, Genoa, Italy

(C) 2020 Association for Computing Machinery.

ACM ISBN 978-1-4503-6861-2/20/07 . \$ \$15.00

https://doi.org/10.1145/3340631.3394875 serving. On the other hand, online ad distribution may be negatively impacted by cannibalization [2, 3, 6, 12, 13], which occurs when two or more competing forces target the same customer base.

Cannibalization comes in a number of forms. Offline-online cannibalization (OOC) is when offline ads cannibalize the market for online ads or vice versa [2, 3, 6]. Typically in OOC, online advertising becomes more important when harder-to-reach users are targeted [6]. Conversely, online advertising is not suitable for introducing products that can fully appeal to users offline [6].Cannibalization between search ads and organic search results (AOC) has also been studied [12,13]. Displaying search ads drives down the display of organic search results, which increases the number of clicks on ads and reduces the number of clicks on search results.

In this paper, we report a new type of cannibalization phenomenon that advertising platform companies encounter, which we call multiple advertisement cannibalization (MAC). Advertising platform companies hold ads in various categories, and users are typically interested in various categories. These categories vary from things that interest a lot of people to things that are interesting to a few. We find that serving a large number of ads in a "minor" category impairs the sales of items in "major" ad categories, even when the targeting is correct in the sense that the served users prefer the minor category. In this case, two competing advertisement categories target the same user base. In other words, it turns out that there is cannibalization, i.e. MAC, in this situation.

In order to overcome MAC, we developed novel user modeling methods and used them to suppress cannibalization. As a result, we increased revenue by $+0.6 \%$ while significantly increasing the impressions (numbers of times the ads has appeared in web sites) of minor category ads.

Our contribution is three-fold:

- We define multiple advertiser cannibalization (MAC), which is a new type of cannibalization problem in online-ad distribution.

- We confirm the existence of MAC through a real ad distribution experiment.

- We propose and validate a method for solving MAC problems with a user modeling technique involving an online questionnaire.

\section{RELATED WORK}

\subsection{User Modeling with Questionnaire}

In recommender systems, it is quite common to model the preferences of new users by conducting short interviews with them [1, $5,7,10,14]$. Blumenstock et al. [4] estimated the interests of users from search logs. This process is often done in active learning [7] or in a bandit setting in which models explore and exploit tradeoffs [11]. The work of Liu et al. [9] is an example of combining 


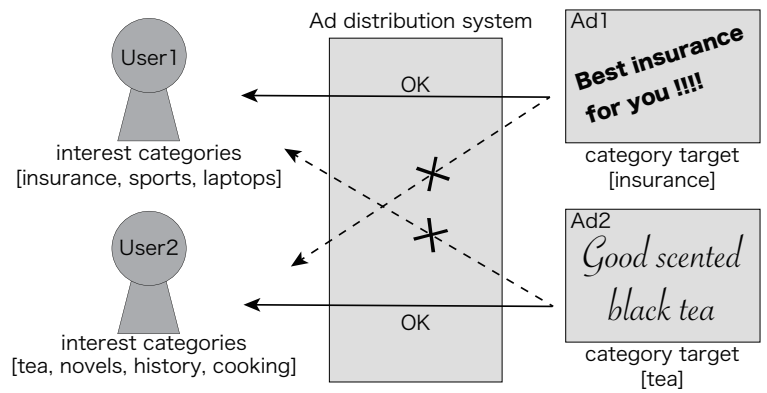

Figure 1: Overview of the category targeting system

questionnaire surveys and digital traces and applying them to a product advertisement recommender system, as in this paper.

\subsection{Cannibalization for online advertising}

Two types of cannibalization in online advertising have been researched: online and offline cannibalization $[2,3,6,8]$ and search ad and organic search cannibalization [12, 13]. Vattikonda et al. [13] presented the first large-scale measurement of effectiveness in terms of incremental conversion gains of online search ads. They describe occurrences of cannibalization in their study that aimed to evaluate an advertiser's strategies. Unlike search ads in [13], our focus is pay-per-click display advertising and to reduce the cannibalization in an advertisement network platform.

\section{PRELIMINARY}

\subsection{Online ad distribution system with category targeting}

The advertisement system divides ads into categories for optimal delivery. The category identifies what kind of user interest an advertisement caters to. On the basis of each user's action history, the system generates a user model with a set of categories the user is interested in and serves ads using this model if the advertisers target their ads to those categories. For example, Ad1 and Ad2 each have "insurance" and "tea" in their target categories, and User1's interest categories include "insurance" but no "tea". In this case, only Ad1 can be delivered to User1; Ad2 cannot be delivered. If another user, User2, has interest categories including "tea" but not "insurance", only Ad2 can be delivered to him or her (Fig. 1 ). After this targeting process, the ads to be delivered are selected by the auction system and bidding begins.

\subsection{Major and minor categories}

It is our experience that major and minor categories naturally arise in ad distribution systems. Ads in major categories are more frequently provided by advertisers than in minor categories and are therefore served and clicked more frequently by users. This imbalance can be expected since there are a large number of ad categories with varying granularity. However, the fact that minor categories are less likely to collect data than major categories hinders the generation of user models for the minor categories and makes them unnecessarily disadvantageous.

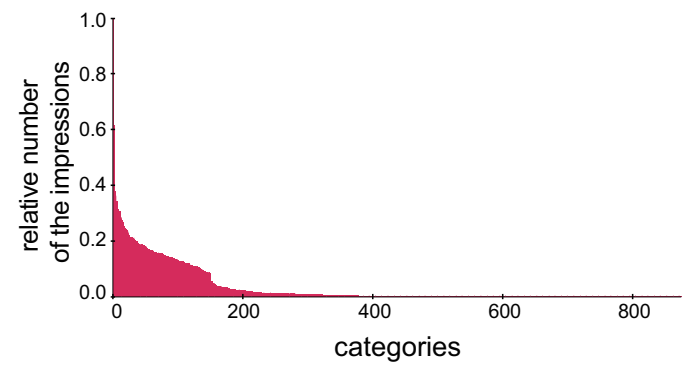

Figure 2: Number of the impressions of each category

On the other hand, in targeting advertisements, it is said that advertisements of minor long-tail products, which are not suited for mass-advertisement, have high appeal to users [6, 8]. Fig. 2 shows the number of impressions of each category in our ad distribution platform. We clearly confirm the long tail phenomenon. To stimulate the market, advertising platform companies have incentives to serve minor ads to users despite having less data on them.

\section{MULTIPLE ADVERTISEMENT CANNIBALIZATION (MAC)}

\subsection{Why does MAC occur?}

Although minor ads are suited for targeting advertisements, delivering too many minor product ads will reduce the exposure of users to major product ads and result in a decrease in total revenue. In this case, minor product ads and major product ads target the same user base, and there are a number of possible reasons for the revenue decrease. One reason may be inaccuracies in the user model. A model for major product ads is likely to be more accurate than one for minor products and often induces more ad clicks, because the abundance of data on major products allows the model to be better trained.

There are various users, from those who are interested in many categories to those who are interested in only a few minor categories. Serving minor-category ads to the latter kind of user is not a problem, but serving them to the former type of user causes competition with other major ads. If we deliver too many ads in the minor category to the former type of user, competition becomes unduly advantageous for the minor category. At the same time, if bids associated with minor-category ads are lower than those of major ads, overall revenue is impaired. This is the problem of MAC.

\subsection{Methods of modeling users to reduce MAC}

To confirm that MAC actually happens, we trained a questionnairebased machine learning model (see section 4.2.1) for minor categories as a baseline at first. Then, we devised two methods to reduce MAC (see section 4.2.2 and 4.2.3).

4.2.1 Questionnaire-based user modeling. We used a machine learning model of users with a questionnaire-based classifier. First, we collected samples that could be used as the ground truth; then, we trained a machine learning model that predicts user behavior. After that, we took an instance (a user) and represented it as a feature vector, where each dimension indicated whether the user searched 
within certain queries in the time period before participating in the questionnaire surveys. We used logistic regression as it is a standard classifier that is easily interpretable. Since it is a linear model, the dimensions that represent the search queries are weighted, and it is possible to extract the queries with a large weight. The hyper parameters of the logistic regression model were optimized through the cross-validation approach. The questions shown below were used to create this model. In the questionnaire, we asked participants to indicate how much you are interested in the goods and services related to the keywords from three viewpoints: Interest, Search behavior, and Purchase experience. To reduce ambiguities and give a context for the keywords, we also provided genre and a subcategory of genre associated with the keywords.

Genre: Entertainment / Movie / Documentary

Keyword: documentary

Q1: Interest- How interested are you in the goods and

services related to this keyword?

Q2: Search behavior- Have you searched for goods or services related to this keyword?

Q3: Purchase experience- Have you purchased goods or services related to this keyword?

The questionnaires included a simple human verification question, and we discarded submissions that failed to answer this question. We identified users who were interested in the keyword as well as had the search behavior or purchase experiences. To create the classifier, we differentiated these users from others on the basis of their answers.

4.2.2 Subtract popular modeling. The first idea is the "SubtractPopular" method. In essence, when a salesperson for the popular categories recommends an expensive item to a customer, a salesperson for the minor categories should not recommend a cheaper item. In this analogy, the items are advertisements and the salesperson is a prediction model. To accomplish this in terms of a linear classifier, we average weight vectors associated with popular categories and subtracted it from each weight vector of a model trained in minor categories. By doing so, we create a prediction model for minor categories, which cannot produce a high score when a competing prediction model for the popular categories produces a high score. To create a feature subtraction model, we collect the ground truth of the users' interests through the online questionnaire survey.

4.2.3 Target silent modeling. The second idea is the "TargetSilent" method, which identifies customers that repeatedly buy a lot of items and excludes those customers when a salesperson for the minor categories recommends an item. Essentially, in this method, we want to target silent users who are unaccustomed to clicking on web ads. To find such users, we asked all users to indicate whether they had a positive or negative attitude toward an advertisement. We trained the model of silent users on the results of those who expressed a negative attitude toward ad clicking; then, we added the estimation score to the minor ad clicking model.

The questionnaire to create TargetSilent model is shown below.

Q1 Do you know that there are ads that are displayed in a format similar to articles like the above?

Q2 How often do you click on ads?
Q3 What do you think about clicking on ads?

Q4 How often do you see ads that fit your interests?

To create the TargetSilent model, we first divided the users into three types. The first type includes users who like to click on advertisements. These users answer (a) in Q2 and answer (a) or (b) in Q3. The second type includes users who are poorly matched. These users answer (a) or (b) in Q3 and answer (c) or (d) in Q4. The third type consists of users who dislike advertisements. These users answer (d) in Q2 and answer (d) in Q3. A model was trained for each user types, and similar to Subtract popular model, we subtracted the weight vector of the first and second and added that of the third model to the base classifier to create TargetSilent model.

\section{EXPERIMENT}

\subsection{Experimental Conditions}

5.1.1 Experimental Overview. The objective of the experiment is twofold: to confirm the existence of MAC in the online ad distribution system, and to evaluate the performance of the proposed methods for preventing MAC. In order to evaluate precisely, we conducted A/B tests for two weeks. We divided the users into two groups (a control group and an experimental group) and the different user modeling methods are applied to each user group. Each group had approximately 73 million unique users in various sites. The ads were divided into 900 categories. After sorting the categories by the number of clicks, about the top $10 \%$ categories were designated as major categories and about the bottom $10 \%$ as minor.

5.1.2 Control Group: Baseline. For the control group, we used a baseline method using manually crafted rules to estimate the users' interests from the search queries. For example, 'Hawaii travel' is manually associated with the ad categories of 'Overseas trip'. The threshold of interest was then determined with logistic regression. For example, the ad category 'Overseas trip' was assigned to any user who searched for 'Hawaii travel' more than five times. While there were about 900 categories, the proposed model and the baseline evaluated only 80 of them (hereafter Target 80 ) with fewer impressions; we confirmed that about less than $1 \%$ of all impressions in the baseline method were from the target categories.

5.1.3 Experimental Group: Comparative methods. The experiments were carried out on the experimental group by replacing the rules based baseline for Target 80 with the proposed model. As for the other categories, the conditions were the same between the control and experimental groups.

We compared the following four models. The BoostMinor model is one which can strengthen the minor category targeting performance without considering MAC. Similar to the proposed user modeling methods, we collected ground truth data through online surveys and found similar users with search queries linked to each. In the BoostMinor model, we focused on increasing the KPIs for the minor categories and did not incorporate any consideration in the MAC problem.

- BoostMinor

- SubtractPopular (See section 4.2.2)

- TargetSilent (See section 4.2.3)

- Both (SubtractPopular + TargetSilent) 


\begin{tabular}{|c|c|c|c|c|}
\hline \multirow{2}{*}{ Target } & Models & \multicolumn{3}{|c|}{ Performance gain } \\
& & impression & iCTR & RPI \\
\hline \multirow{5}{*}{ Target 80} & BoostMinor & $+1072.16 \%$ & $+22.88 \%$ & $+5.77 \%$ \\
& SubtractPopular & $+135.33 \%$ & $+6.46 \%$ & $+3.04 \%$ \\
& TargetSilent & $+242.46 \%$ & $+3.16 \%$ & $+1.20 \%$ \\
& Both & $+160.87 \%$ & $+4.53 \%$ & $+0.15 \%$ \\
\hline \multirow{5}{*}{ Others } & BoostMinor & $-6.19 \%$ & $-0.38 \%$ & $-0.87 \%$ \\
& SubtractPopular & $-1.50 \%$ & $-0.23 \%$ & $-0.13 \%$ \\
& TargetSilent & $-2.65 \%$ & $-0.26 \%$ & $-0.11 \%$ \\
& Both & $-1.65 \%$ & $-0.22 \%$ & $+0.31 \%$ \\
\hline \multirow{5}{*}{ All } & BoostMinor & - & $-0.10 \%$ & $-0.05 \%$ \\
& SubtractPopular & - & $-0.02 \%$ & $+0.20 \%$ \\
& TargetSilent & - & $+0.01 \%$ & $+0.43 \%$ \\
& Both & - & $-0.04 \%$ & $\mathbf{+ 0 . 6 2 \%}$ \\
\hline
\end{tabular}

Table 1: Evaluation results

5.1.4 Evaluation metrics. We evaluated the performance of the user modeling methods by partitioning the interest categories into Target 80 and "Others", as described above. To evaluate the models, we utilized three evaluation metrics, i.e., impression, impression click through rate (iCTR), and revenue per impression (RPI). The impression score shows how many advertisements were shown to users. The iCTR score shows how many advertisements were clicked among the total impressions of the advertisements shown to the users. The RPI score shows the estimated earnings of the algorithms among the total impressions. Each metric is represented by the gain in KPI score of the proposed method against that of the baseline model.

\subsection{Experimental Results}

Table 1 denotes the percentage gains of each method against the baseline rules-based method. Before confirming the performance of the proposed methods, we confirmed whether MAC actually occurred or not.

5.2.1 Existence of MAC. The "BoostMinor" rows show the results of the user model that collects ground truth data of the minor categories from the online questionnaire survey method; this method does not consider cannibalization. As shown, while impression, iCTR and RPI dramatically improved for Target 80 , the interest categories in Others performed much worse compared with the existing baseline method. As these categories hinder overall performance, both iCTR and RPI for all categories (see All) are worse compared with those of the baseline method. Since it is the overall performance that counts, the "BoostMinor" method is not a satisfactory replacement for the baseline method. A cannibalization problem occurred, in which users who would have clicked advertisements in popular categories (interest categories in Others) actually clicked advertisements in minor categories (Target 80 ) instead.

In the "BoostMinor" rows, there is a lower overall iCTR than the baseline, and this indicates that a number of such transfers were performed incorrectly. Even when the transfers did not affect iCTR, that is, the users were likely to click both advertisements from the Target 80 and Others categories, they could affect RPI, since the revenue from clicking popular categories tended to be higher than that from clicking minor categories. Figuratively speaking, this situation is much like selling a cheaper item when a customer is willing to buy an expensive item. We see that it is difficult to offset the loss from the popular categories with the gain in revenue from the minor categories, and the total revenue decreases as a result of cannibalization. Whenever new changes take place, business processes involving markets that operate frequently could face the cannibalization problem. Thus, we need to have a global perspective when tuning part of a system such as the model of Target 80 .

5.2.2 Performance of the proposed user modeling methods to solve $M A C$. The impression, iCTR, and RPI columns in the "SubtractPopular", "TargetSilent" and "Both" rows show negligible cannibalization between popular "Others" and minor "Target 80" categories. We thus accomplished the dual objective of improving the impression of minor categories without hurting popular categories and improved the overall RPI of the proposed method. Thus, we were able to solve the MAC problem.

In the SubtractPopular, TargetSilent and Both rows, we note that for categories that belong to the Others column, iCTR is about $-0.2 \%$, but RPI is not as low. The difference is due to the cost per click (CPC), which is the amount used to bid on advertisements. We observed that CPC increased for Others, and as RPI is calculated as $i C T R \times C P C$, RPI was not as low as predicted due to the decrease in iCTR. There could be several reasons for this; we postulate two of them as follows. First, as the impressions for Others went down, the overall advertisements in these categories became harder to display. As a result, it could be that only high-bid advertisements ranked high in the inventory were served and low-bid advertisements were less likely to be served. The other reason is that the matching system for Others had adapted to a reduced number of users with fewer behavioral variations. This may have enabled the system for Others to match users with advertisements with high bids more effectively, pushing up the CPC. Similarly, for the Target 80 group, the performance gain for iCTR is larger than that for RPI. As the impressions for categories in Target 80 increased, the inventory of highly bid advertisements in these categories became smaller. Also, we postulate that the proposed "Both" method that combines SubtractPopular and TargetSilent targeted users that are more difficult to match with high-bid advertisements, who may not show much interest in advertisements in general. By targeting users who are especially essential for minor Target 80 categories and are not for major Others categories, the proposed "Both" method was able to increase the impression in Target 80 and RPI in both Target 80 and Others while suppressing excessive reduction in the impressions from users who tend to click high CPC ads in Others.

\section{CONCLUSION}

The existence of MAC, a new cannibalization problem for online advertising platform companies, is confirmed in this paper. Moreover, we also confirmed the MAC causes deterioration of total performance, and thus MAC is a serious issue for advertising platform companies. In order to solve it, we developed two user modeling methods: SubtractPopular and TargetSilent model. Experimental results indicated that these user models help to prevent MAC and improve the overall performance of ad distribution. 


\section{REFERENCES}

[1] Michal Aharon, Oren Anava, Noa Avigdor-Elgrabli, Dana Drachsler-Cohen, Shahar Golan, and Oren Somekh. 2015. ExcUseMe: Asking Users to Help in Item Cold-Start Recommendations. In Proceedings of the 9th ACM Conference on Recommender Systems (RecSys '15). ACM, New York, NY, USA, 83-90. https://doi.org/10.1145/2792838.2800183

[2] Susan Athey and Joshua Gans. 2010. The Impact of Targeting Technology on Advertising Markets and Media Competition. Joshua S Gans 100 (01 2010) https://doi.org/10.2139/ssrn.1535325

[3] Dirk Bergemann and Alessandro Bonatti. 2012. Targeting in Advertising Markets: Implications for New and Old Media. (12 2012).

[4] Joshua Blumenstock, Gabriel Cadamuro, and Robert On. 2015. Predicting poverty and wealth from mobile phone metadata. Science 350 (11 2015), 1073-1076. https://doi.org/10.1126/science.aac4420

[5] Nadav Golbandi, Yehuda Koren, and Ronny Lempel. 2011. Adaptive Bootstrapping of Recommender Systems Using Decision Trees. In Proceedings of the Fourth ACM International Conference on Web Search and Data Mining (WSDM '11). ACM, New York, NY, USA, 595-604. https://doi.org/10.1145/1935826.1935910

[6] Avi Goldfarb and Catherine Tucker. 2011. Search engine advertising: Channel substitution when pricing ads to context. Management Science 57, 3 (2011), 458-470.

[7] Abhay S. Harpale and Yiming Yang. 2008. Personalized Active Learning for Collaborative Filtering. In Proceedings of the 31st Annual International ACM SIGIR Conference on Research and Development in Information Retrieval (SIGIR '08). ACM, New York, NY, USA, 91-98. https://doi.org/10.1145/1390334.1390352

[8] Mark Hart. 2007. The Long Tail: Why the Future of Business Is Selling Less of More by Chris Anderson. Journal of Product Innovation Management 24 (04 2007),
274 - 276. https://doi.org/10.1111/j.1540-5885.2007.00250.x

[9] Zhe Liu, Yi Wang, Jalal Mahmud, Rama Akkiraju, Jerald Schoudt, Anbang Xu, and Bryan Donovan. 2016. To Buy or Not to Buy? Understanding the Role of Personality Traits in Predicting Consumer Behaviors. 337-346. https://doi.org/ 10.1007/978-3-319-47874-6_24

[10] Seung-Taek Park, David Pennock, Omid Madani, Nathan Good, and Dennis DeCoste. 2006. NaiVe Filterbots for Robust Cold-start Recommendations. In Proceedings of the 12th ACM SIGKDD International Conference on Knowledge Discovery and Data Mining (KDD '06). ACM, New York, NY, USA, 699-705. https: //doi.org/10.1145/1150402.1150490

[11] Tobias Schnabel, Paul N. Bennett, Susan T. Dumais, and Thorsten Joachims. 2018. Short-Term Satisfaction and Long-Term Coverage: Understanding How Users Tolerate Algorithmic Exploration. In Proceedings of the Eleventh ACM International Conference on Web Search and Data Mining (WSDM '18). ACM, New York, NY, USA, 513-521. https://doi.org/10.1145/3159652.3159700

[12] Greg Taylor. 2012. Search Quality and Revenue Cannibalisation by Competing Search Engines. Fournal of Economics Management Strategy 22 (03 2012). https: //doi.org/10.1111/jems.12027

[13] Bhanu C Vattikonda, Vacha Dave, Saikat Guha, and Alex C Snoeren. 2015. Empirical analysis of search advertising strategies. In Proceedings of the 2015 Internet Measurement Conference. ACM, 79-91.

[14] Ke Zhou, Shuang-Hong Yang, and Hongyuan Zha. 2011. Functional Matrix Factorizations for Cold-start Recommendation. In Proceedings of the 34th International ACM SIGIR Conference on Research and Development in Information Retrieval (SIGIR '11). ACM, New York, NY, USA, 315-324. https://doi.org/10.1145/2009916. 2009961 